\title{
Pancreatic acinar cell carcinoma presenting as a third metachronous primary tumor with unconventional features
}

\author{
Jennifer Ford ${ }^{1}$, Ghassan Tranesh ${ }^{2 *}$, Paul Mazzara ${ }^{3}$, Mohammed Barawi ${ }^{4}$, Jimmy Haouilou ${ }^{5}$ and Richard Berri' \\ ${ }^{1}$ Department of Surgery, St. John Hospital and Medical Center, Detroit, MI, USA \\ ${ }^{2}$ Department of Pathology, Mayo Clinic, Jacksonville, FL, USA \\ ${ }^{3}$ Department of Pathology, St. John Hospital and Medical Center, Detroit, MI, USA \\ ${ }^{4}$ Department of Gastroenterology and Endoscopy, St. John Hospital and Medical Center, Detroit, MI, USA \\ ${ }^{5}$ Department of Vascular surgery St. John Hospital and Medical Center, Detroit MI, USA \\ ${ }^{6}$ Section of Surgical Oncology, St. John Hospital and Medical Center, Detroit, MI, USA
}

\begin{abstract}
Acinar cell carcinoma (ACC) of the pancreas is a rare neoplasm, accounting only 1-2\% of pancreatic cancers, and is characterized by exhibiting exocrine differentiation and lacking significant ductal or endocrine differentiation. A diagnostic difficulty exists in that presenting symptoms and conventional radiologic findings in these patients are usually non-specific. Owing to high propensity for vascular and perineural invasion, the majority of the patients with ACC have metastatic disease at presentation, resulting in poor prognosis even after curative surgical resection.

A 60-year-old male presented at our institution only complaining of vague left upper quadrant abdominal pain. Notably, his past medical history was significant for thyroid and prostate cancers. Other than slightly elevated serum lipase level (58 U/L), his preoperative laboratory data were unremarkable. Initial computerized tomography $(\mathrm{CT})$ scan revealed a well-defined solitary mass $(4 \mathrm{~cm} \times 3.4 \mathrm{~cm} \times 1.7 \mathrm{~cm})$ in the junction of the pancreatic neck and body, which was confirmed by subsequent endoscopic ultrasound (EUS), depicting a $3 \mathrm{~cm}$ hypoechoic homogeneous mass. Upon exploration, it was discovered that the tumor had invaded posteriorly into the splenic artery at its origin, splenic vein and portal confluence. Accordingly, the patient underwent distal pancreatectomy and splenectomy with curative intent.

The final histopathologic studies on the surgical specimen revealed an uncommon mixed acinar-ductal differentiation pattern. In addition, perineural invasion was noted, while commonly observed angiolymphatic invasion was absent. Contrary to conventional immunohistochemical diagnostic criteria for ACC, the neoplastic cells were immunoreactive with CD10 and negative for keratin. Other than these unusual features, our case fulfilled the immunohistochemical diagnosis of ACC. The American Joint Committee on Cancer (AJCC) pathologic stage determined for this case was T3N0Mx.

This case is unique clinically and immunohistochemically. One of the peculiar yet intriguing clinical features was its presenting as a third metachronous primary tumor. Most interestingly, there was no evidence of metastatic disease despite the presence of vascular and perineural invasion, which are thought to influence the disease-free survival and recurrence significantly. Its unconventional staining patterns also made immunochemical diagnosis of this case challenging.
\end{abstract}

\section{Introduction}

An estimated 43,920 newly diagnosed cases of primary pancreatic cancer are expected to occur in the United States in 2012 [1]. Of those, Pancreatic Acinar cell carcinoma (ACC) accounts for $<1-2 \%$ of primary tumors of the exocrine pancreas [2-4]. Although the normal function of acinar cells has long been understood, this neoplasm is poorly defined and underappreciated. Acinar cell tumors are highly malignant with a predisposition to recur [3]. Owing to high propensity for vascular, angiolymphatic and perineural invasion, metastatic disease will be present in more than half of patients at the time of presentation [3]. Overall, prognosis is marginally elevated over that of a ductal adenocarcinoma but inferior to that of pancreatic neuroendocrine neoplasms [5-8].

Typically seen is a male predominance during the fifth to seventh decades of life [2-4].

The most common presentation is that of nonspecific symptoms: weight loss, fatigue and abdominal pain. Schmid's Triad (a combination of subcutaneous fat necrosis, polyarthralgia and eosinophila) or hormone-specific endocrine symptoms have also been reported $[2,3,9]$.

National Comprehensive Cancer Network (NCCN) protocol requires a specialized computerized tomography (CT) or magnetic resonance imaging (MRI) upon discovery of a pancreatic mass suspicious of malignancy [10]. Optimal multi-phase imaging techniques include a non-contrast, arterial, parenchymal and portal venous phases of contrast enhancement captured in $3 \mathrm{~mm}$ cross sections through the abdomen [10]. ACCs have been reported in the head, uncinate process and tail of the pancreas. A Positron emission tomography (PET) scan may be considered as adjunct to a formal pancreatic CT protocol in high-risk patients with metastasis [10]. On

Correspondence to: Ghassan Tranesh, Department of Pathology, Mayo Clinic, Jacksonville, FL, USA, E-mail: tranesh@gmail.com

Received: January 07, 2016; Accepted: January 23, 2016; Published: January 26, 2016 
both imagine modalities, ACCs are most often found to be a welldefined solitary mass appearing heterogeneous and hypovascular with a well-defined enhanced pseudocapsule [11,12]. Radiologic differential diagnosis includes ductal adenocarcinoma, neuroendocrine tumor, solid and pseudopapillary tumor, pancreatoblastoma, mucinous cystic neoplasm and pseudocyst [3].

To determine surgical candidacy a multidisciplinary analysis, endoscopic ultrasonography (EUS) with biopsy, liver function tests, chest CT, carbohydrate antigen (CA) 19-9 levels and a diagnostic laparoscopy should be obtained [10].

There is no grading or staging system specific for ACCs; the American Joint Committee on Cancer (AJCC) staging established for ductal adenocarcinoma is applied $[10,13]$. In accordance with the AJCC, resectable tumors should demonstrate no distant metastases, no radiographic evidence of superior mesenteric vein (SMV) and portal vein abutment, distortion, tumor thrombus, or venous encasement, clear fat planes around the celiac axis, hepatic artery, and superior mesenteric artery (SMA) [10].

Morphologic features include pleomorphic, round basal nuclei with increased mitotic figures in single large central vesicular nucleoli. Cytoplasm typically stains eosinophilc with granular characteristics [13]. Gross arrangement is in lobular patterns without desmoplastic stroma [13].

Immunohistochemical (IHC) staining provides the definitive diagnosis [2,3]. A positive stain for trypsin and chymotrypsin is confirmatory of the diagnosis in $90 \%$ of cases [2,4]. In addition, lipase, keratin and alpha-feto-protein (AFP) are measured $[2,4,13]$. Other IHC features include granular Periodic Acid-Schiff $\mathrm{d}+(\mathrm{PASd}+)$ cytoplasm, beta-catenin/cluster of differentiation (CD)56/CD10 negative, alpha 1 antitrypsin negative, keratin positive, $C D 99$ negative and diastase resistance [13]. Synaptophysin and chromogranin A stain positive in $30-50 \%$ of cases and should more than $25 \%$ of the tumor stain positive, criteria for a mixed acinar-endocrine tumor $[2,13]$. Immunohistochemical staining of mixed acinar cell-endocrine tumors can identify insulin, glucagon and/or somatostatin depending on the endocrine-specific nature of the neoplasm [2].

A 60-year-old male presented at our institution complaining only of vague left upper quadrant abdominal pain. Notably, his past medical history was significant for thyroid and prostate cancers. Other than slightly elevated serum lipase level ( $58 \mathrm{U} / \mathrm{L}$ ), his preoperative laboratory data were unremarkable, including those for any tumor markers. CT scan revealed a well-defined solitary mass $(4 \mathrm{~cm} \times 3.4 \mathrm{~cm} \times 1.7 \mathrm{~cm})$ in the junction of the pancreatic neck and body, which was confirmed by subsequent EUS, depicting a $3 \mathrm{~cm}$ hypoechoic homogeneous mass. Upon exploration, it was discovered that the tumor had invaded posteriorly into the splenic artery at its origin, splenic vein and portal confluence. Despite the tumor extension into the SMV, there was no lumen narrowing. Furthermore, the right side of the SMV was free of tumor with no evidence of spread. Accordingly, the patient underwent distal pancreatectomy and splenectomy with curative intent. The patient had a brief uneventful recovery.

\section{Case report}

A 60-year-old male with significant past medical history of primary prostate cancer and primary thyroid cancer was referred to surgical oncology due to a pancreatic mass. The initial computed tomography (CT) scan revealed a suspicious mass measuring $3.5 \mathrm{~cm} \times 1.8 \mathrm{~cm}$ in the proximal pancreatic body. There was no evidence of vascular involvement or adenopathy. At the primary clinic visit, the patient was counseled on the likely malignant nature of this mass and the pancreatic cancer protocol was initiated.

Repeat CT scan performed in accordance to pancreatic cancer protocol stipulations revealed a heterogeneous mass, suspicious of a primary malignancy at the junction of the body and neck of the pancreas. Radiographically it measured at $4 \mathrm{~cm} \times 3.4 \mathrm{~cm} \times 1.7 \mathrm{~cm}$ with a $1.7 \mathrm{~cm}$ necrotic or cystic component inferiorly. The mass marginated the splenic and portal vein confluence with approximately $180^{\circ}$ circumferential contact. Involvement of the splenic artery could not be excluded, but arterial and venous flow was preserved. There was no involvement of the superior mesenteric artery or celiac axis (Figures 1 and 2). No adenopathy, ascites, or peritoneal masses were reported. Preoperative CA19-9 level was 15.9, carcionembryonic (CEA) 1.2, Lipase 58 and Amylase 89.

An esophagogastroduodenoscopy (EGD) was unremarked but an endoscopic ultrasound (EUS) revealed a $3 \mathrm{~cm}$ hypoechoic, homogeneous mass in the neck of the pancreas that involved the splenic artery. The main pancreatic duct was dilated at $2.8 \mathrm{~mm}$ diameter. An EUS-guided fine needle aspiration biopsy confirmed a pancreatic adenocarcinoma.

Laparoscopic viewing of the abdomen revealed no metastatic disease and open resection proceeded. The lesser sac was entered, the entire right transverse colon and splenic flexure taken down, mobilized and packed into the lower abdomen. The duodenum was completely kocharized and once all short gastric arteries were taken

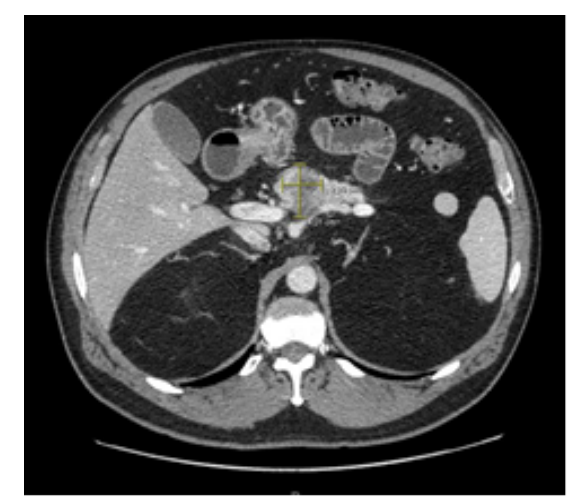

Figure 1. CT scan, axis 30/60/90 using water omin $350125 \mathrm{cc}$ contrast consistent with pancreatic protocol. The pancreatic mass can be appreciated in the center field of view measuring $2.99 \times 4.01 \mathrm{~cm}$.

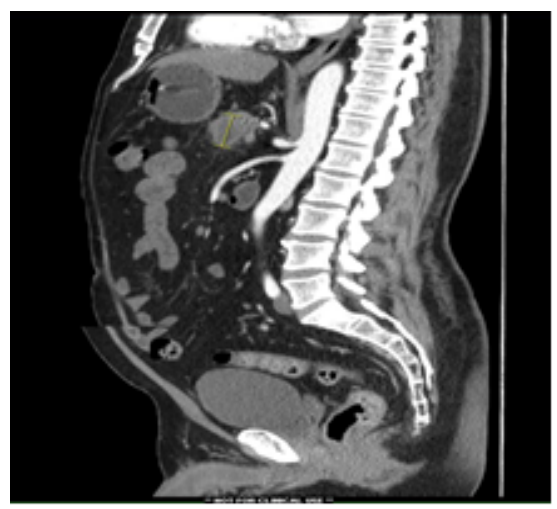

Figure 2. Sagittal CT scan, axis 30/60/90 using water omin $350125 \mathrm{cc}$ contrast consistent with pancreatic protocol. The pancreatic mass can be appreciated in the center field of view measuring $2.99 \times 4.01 \mathrm{~cm}$. 
down, a large tumor in the body of the pancreas was palpated. The tumor appeared to invade posterior and extended into the splenic vein and SMV confluences. A plane of dissection was established inferior to the neck of the pancreas, which allowed direct visualization the superior mesenteric vein (SMV) from the right; no signs of invasion were present. However, the left border of the SMV and splenic vein appeared to be adherent with the tumor. Dissection of the celiac axis demonstrated that the tumor had invaded the splenic artery at the take off from the celiac axis. The celiac axis, hepatic artery and mesenteric artery remained clear of tumor and resection proceeded.

All splenic attachments were taken down and it was mobilized medially to allow access to the posterior retroperitoneal plan. Dissection continued with relative ease; the spleen and pancreatic tail were freed and suspended in a vertical fashion. Apparent at that time, was tumor invasion posteriorly into the splenic vein, splenic artery and confluence of the SMV. At this point, an intra-operative vascular surgery consult was ordered.

The two vascular surgeons identified the origin of the splenic and transected it in close proximity to the celiac axis. The splenic vein, SMV and the confluence were identified; tumor involved the SMV and splenic vein only. Soft pancreas was palpated to the right of the SMV and so chosen for the site of transection. The pancreas was transected and suture ligation in 3 quadrants: the longitudinal, superior and inferior aspects. A vascular clamp was then placed on the SMV and a knife was used to transect the vessel freeing the entire pancreatic body, neck and tumor with the tail and spleen en bloc resection. The splenic vein and SMV confluence was over sewn with a running prolene stitch; flow was patent and hemostasis was excellent.

On the transected surface of the pancreas, the main pancreatic duct identified, dilated and ligated with two prolene sutures. The pathologist indicated that the pancreatic margins, taken from the right of the SMV, were free of tumor. The cut edge of the pancreas, was oversewn and a portion of the omentum was brought up and attached.

At this point the entire abdomen was explored and found to be free of any abnormalities. The abdomen was closed, a 19-French JacksonPratt (JP) drain was placed in the left-upper-quadrant/splenic bed and secured. The patient had a noncomplicated post-operative course and was discharged to home on post-operative day eight.

The final pathologic study of the resected specimen revealed a tumor $3.9 \mathrm{~cm}$ in size with perineural invasion and negative angiolymphatic invasion. Final staging, according to American Joint Committee on Cancer (AJCC) was pT3 N0. The neoplastic cells exhibited a ductal pattern, but were primarily acinar in morphology. Cells were focally immunoreactive with CA 19-9 and exhibited a canalicular pattern of staining with CD10. The cells stained positive for cytokeratin 7 and cytokeratin cam 5.2. The specimen was sent to an outside facility for second opinion; it was diagnosed and confirmed as acinar cell tumor. Noted also was the infiltrative nature of this tumor which may result in a more aggressive nature than most acinar cell tumors.

\section{Discussion}

Acinar cell carcinomas (ACC) of the pancreas are rare and poorly understood; the limited number of literature reviews and case reports fail to identify definitive prognosis indicators and effective treatment modalities. Commonly acinar cell prognosis is superior to that of a ductal carcinoma, but poorer than endocrine pancreatic tumors [2]. Survival ranges between 5 and 38 months; mean post operative disease free period for resectable tumors is approximately 9 months $[2,14]$.
A study conducted by Butturini et al reported $100 \%$ recurrence rates at a median follow up time of 2.6 years (31.4 months) [3]. Toll et al. reported post-operative survival rates at one, three and five years of: $57 \%, 26 \%$ and $6 \%$ [4]. Metastatic disease will be present in more than half of patients at the time of presentation and diagnosis. Upwards of $25 \%$ develop metastatic disease at a later date [3]. Most common sites of metastasis include the liver and regional lymph nodes [4]. Features associated with a poorer prognosis included size $(>6.5 \mathrm{~cm})$, positive lymph nodes, distant metastasis and advanced staging [14]. The patient presented possessed a mirad of prognostic indicators: advanced stage (pT3), lack of metastasis (local or distant) and size $<6.5 \mathrm{~cm}$.

This rare neoplasm has a peak incidence in the fifth to seventh decade of life, with a male (2:1) predominance [2-4]. A presentation of subcutaneous fat necrosis, polyarthralgia and eosinophila, deemed "Schmid's Triad," is consider almost pathognomonic for ACCs but the incidence remains low $[2,3,9]$. Schmid's Triad is due to increased serum lipase secreted by the tumor $[2,3]$. Some also present with mixed endocrine symptoms, specific to the tumor endocrine differentiation $[2,3,9]$. However, most cases including the patient presented here, present with nonspecific symptoms: weight loss, abdominal pain, adding to the diagnostic difficulty of ACCs.

Radiographic diagnosis is difficult due to similar nonspecific findings among multiple pancreatic neoplasms. Ductal adenocarcinoma, ACCs (including the case presented here), neuroendocrine tumors, solid and pseudopapillary tumors and pancreaticobalstoma all appear with a well-defined solitary mass appearing heterogeneous and hypovascular with a well-defined enhanced capsule $[2,3,11,12]$. The presence of a necrotic or cystic component, such as this case, can further widen the radiographic differential diagnosis to include mucinous cystic neoplasm and pseudocysts.

An endoscopic ultrasound (EUS) with fine-needle-aspiration biopsy can help different the radiographic findings. In the case presented, the biopsy result resulted in a non-specific pre-operative diagnosis of adenocarcinoma.

No resection guidelines specific for ACCs exist; surgical respectability, the treatment of choice determined by pre-operative imaging, follows ductal carcinoma guidelines [2,3,10]. Surgical resection is associated with an increase in mean survival months [3]. Table 1 details findings by Butturini et al. (2011) and the associated increase in survival months after resection. Butturini also reported at their center, The Verona Center, post-resection two of nine patients were alive without disease at 85 months (status post distal pancreatectomy) and the other alive with disease at 52 months (status post pylorus-preserving pancreatic duodenectomy) [3]. Both resections revealed T2N0MX disease. Following AJCC guidelines, final staging for the case presented here was determined to be pT3N0MX and due to its more extensive invasiveness, this may relate to a poorer prognosis that that seen at the Verona Center.

Final pathologic diagnosis may demonstrate features of ductal or endocrine carcinoma resulting in a mixed acinar-ductal or acinarendocrine tumor $[4,14]$. Upwards of $44 \%$ of ACCs have concomitant endocrine neoplastic features. It was thought that mixed tumors have an improved morbidity and mortality rate, comparative to typical ACCs [15-17]. However, a recent study by La Rosa et al. reported no difference in survival between ACC and mixed ACC-endocrine carcinomas [14]. Final pathology sent from our specimen was observed to have a mixed ductal component; a finding reported less than mixed endocrine features. This particularly rare combination of acinar-ductal 
Table 1. Selected literature series of ACCs; modified from Buttirini et al., 2011.

\begin{tabular}{|c|c|c|c|c|c|}
\hline Author (year) & No. of patients & No of resections & Other treatment & Median Survival (months) & Notes \\
\hline Holen (2002) & 39 & 18 & $\begin{array}{l}2 \text { neoadjuvant therapy } \\
12 \text { nonresectable }\end{array}$ & $\begin{array}{l}36 \text { (resectable group) } \\
19 \text { (nonresectable) }\end{array}$ & \multirow{2}{*}{$\begin{array}{c}2 \text { pancreatoblastomas; } 9 \text { patients no treatment } \\
\text { started } \\
2 \text { mixed acinar-endocrine tumors }\end{array}$} \\
\hline Matos (2009) & 17 & 13 & 1 neoadjuvant therapy & $\begin{array}{l}61 \text { (resectable group) } \\
39 \text { (nonresectable) }\end{array}$ & \\
\hline Schmidt (2008) & 865 & 333 & Adjuvant therapy in $42.9 \%$ & $\begin{array}{l}27 \text { (resectable group) } \\
7 \text { (non resectable) }\end{array}$ & \\
\hline Wisnoski & 672 & 266 & None stated & $\begin{array}{c}127 \text { (resectable) } \\
47 \text { (nonresectable) }\end{array}$ & Inclusion of neuroendocrine tumors in study \\
\hline
\end{tabular}

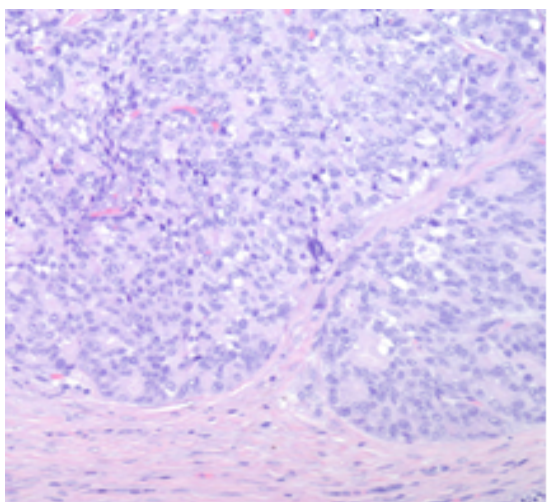

Figure 3. Low power magnification of the pancreas demonstrating a desmoplastic reaction interspersed between sheets and islands of acinic cell carcinoma.

carcinoma is reported to have a similar prognosis to that of pure ACC that remains improved from pure ductal adenocarcinoma [2-4,14].

Morphologically, ACCs are often highly cellular with minimal stroma and no desmoplastic stromal response [13,18,19]. Most neoplasms are solid, but cystic subtypes have been reported [3]. Microscopically, there are four patterns of histologic growth: acinar, cellular, trabecular and glandular $[9,13,20]$. Typical histologic features include solid sheets of hyperchromic, round nuclei surrounded by eosinophilic cytoplasm in a polarized arrangement $[2,4,13]$. Figure 3 demonstrates a low power magnification of our specimen. It demonstrates a desmoplastic reaction interspersed between sheets and islands of acinic cell carcinoma. This morphologic finding challenges yet another aspect of ACCs that was previous agreed upon: no desmoplastic response.

Immunohistochemical (IHC) staining provides the definitive diagnosis [2,3]. Diagnostic staining for ACCs include positive lipase, chymotrypsin, trypsin and kertain with variable alpha-feto-protein (AFP) $[2,4,13]$. A positive stain for trypsin and chymotrypsin is confirmatory of the diagnosis in $90 \%$ of cases [2,4]. A 2012 study by La Rosa et al challenged this and stated that B-Cell lymphoma/ leukemia 10 (BCL 10) and trypsin where the most reliable markers, while amylase and lipase were least reliable for diagnosis [14]. The case presented here was negative for keratin, yet the positive for cytokeratin 7 and cytokeratin cam 5.2, which is unique, and to our knowledge not yet reported elsewhere (Figure 4). Other general IHC features include granular PASd+ cytoplasm, beta-catenin/CD56/CD10 negative, alpha 1 antitrypsin negative, CD 99 negative and diastase resistance [13]. Yet another feature adding to the uniqueness of this case is the positive canalicular pattern of staining with CD 10.

Traditionally, typical genetic mutations present in pancreatic adenocarcinomas such as k-ras, p53, were thought to not be common

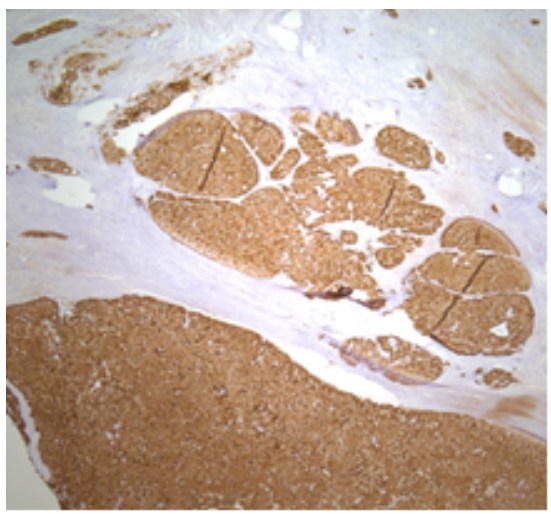

Figure 4. A medium power magnification of the pancreas demonstrating acinic cell carcinoma with a strong positive cytokeratin 7 immunostain.

in ACCs $[3,4]$. Relations to the Adenamatous polyposis coli (APC)/ Beta-catenin mutation pathway had been theorized [3,4]. However, a recent article by B Kong et al. demonstrated development of a murine tumor, similar to that of a human ACC, when hyperactive mammalian target of rapamycin (mTOR) was combined with loss of p53 [21]. They demonstrated, in the presence of hyperactive mTOR signaling, the ablation of $\mathrm{p} 53$ programmed cell death promoted dysplastic acinar cells and ultimately a malignancy resembling ACC [21]. Even mice with a premature demise (due to uncontrolled diabetes mellitus) showed evidence of precancerous lesions within their pancreas [21]. Although a formal relationship has yet to be established, this study demonstrates consistent evidence that mTOR-p53 is potentially an integral part in the ACC genetic model.

Neoadjuvant chemoradiation can be used in borderline resectable and locally advanced disease [3]. However, it is still unknown which regimens provide the most benefit. Many different chemotherapeutic regimens have been investigated: 5-fluorouracil, gemcitabine, leucovorin and Irinotecan are often used and thought effective due to the relation with APC/Beta-catenin pathway (known in colon cancer and suspected in ACC) $[2,3]$. Other combinations can include docetaxel, capecitabine and oxaliplatine [3]. Experimental agents include TS-1 (5-chloro-2,4-dihydroxyuridine and potassium oxonate) and cisplatin I [2]. These agents are suspected to be successful due to the similarities and possible common molecular genetics between ACC and pancreaticoblastoma [22-26]. Although it is suspected that the use of chemoradiation and neoadjuvant chemoradiation may increase survival months, the limited number of case studies fails to identify a definitive survival benefit or combination that which significantly correlates to an increase in survival.

In summary, our case demonstrated a variant of ACC, not otherwise reported: a third primary malignancy with positive cytokeratin and CD10 staining. This case highlights the variability of acinar cell 
carcinomas and draws attention to the need for further understanding of the neoplasm prognostically, morphological, imunohistochemically and genetically. At the time of submission, approximately nine months post-operatively, the patient remains disease free. This draws into question the reliability of the agreed upon prognostic characteristics of ACC. Currently, surgical resection is the only method demonstrated to moderately increase survival. However, survival rates remain poor after resection with a lack of standardized neoadjuvant chemoradiation treatment. A deeper morphologic and genetic understanding of this neoplasm would allow researchers to select more targeted chemotherapy agents with hopes of decreasing recurrence rates and increasing survival time.

\section{Acknowledgement}

Special thanks to: William Yoon, Ambar Banerjee and Kumara Rama from the Department of Surgery, St. John Hospital and Medical Center, Detroit, MI, USA who assisted the case intra-operatively and Joel Greenson from the Department of Pathology, University of Michigan, Ann Arbor, MI who provided a second and confirmatory pathologic diagnosis.

\section{References}

1. Landis MD, Lehmann BD, Pietenpol JA, Chang JC (2013) Patient-derived breast tumor xenografts facilitating personalized cancer therapy. Breast Cancer Res 15: 201. [Crossref]

2. Antoine M, Khitrik-Palchuk M, Saif MW (2007) Long-term survival in a patient with acinar cell carcinoma of pancreas. A case report and review of literature. JOP 8: 783789. [Crossref]

3. Butturini G, Pisano M, Scarpa A, D'Onofrio M, Auriemma A, et al. (2011) Aggressive approach to acinar cell carcinoma of the pancreas: a single-institution experience and a literature review. Langenbecks Arch Surg 396: 363-369. [Crossref]

4. Toll AD, Mitchell D, Yeo CJ, Hruban RH, Witkiewicz AK (2011) Acinar cell carcinoma with prominent intraductal growth pattern: case report and review of the literature. Int J Surg Pathol 19: 795-799. [Crossref]

5. Tanaka M, Chari S, Adsay V, Fernandez-del Castillo C, Falconi M, et. al (2006) International consensus guidelines for management of intraductal papilarry mucinous neoplasm and mucinous cystic neoplasm of the pancreas. Pancreatology 6: 17 . [Crossref]

6. Wisnoski NC, Townsend CM Jr, Nealon WH, Freeman JL, Riall TS (2008) 672 patients with acinar cell carcinoma of the pancreas: a population-based comparison to pancreatic adenocarcinoma. Surgery 144: 141-148. [Crossref]

7. Schmidt CM, Matos JM, Bentrem DJ, Talamonti MS, Lillemoe KD, et al. (2008) Acinar cell carcinoma of the pancreas in the United States: prognostic factors and comparison to ductal adenocarcinoma. J Gastrointest Surg 12: 2078-2086. [Crossref]

8. Holen KD, Klimstra DS, Hummer A, Gonen M, Conlon K, et al. (2002) Clinical characteristics and outcomes from an institutional series of acinar cell carcinoma of the pancreas and related tumors. J Clin Oncol 20: 4673-4678. [Crossref]

9. Aqel B, Scolapio J, Nguyen J, Krishna M, Raimondo M (2004) Recurrent pancreatitis due to a cystic pancreatic tumor: a rare presentation of acinar cell carcinoma. JOP 5: 151-154. [Crossref]
10. http://www.nccn.org/professionals/physician_gls/f_guidelines.asp

11. Tatli S, Mortele KJ, Levy AD, Glickman JN, Ros PR, et al. (2005) CT and MRI features of pure acinar cell carcinoma of the pancreas in adults. AJR Am J Roentgenol 184: 511-519. [Crossref]

12. Chiou YY, Chiang JH, Hwang JI, Yen CH, Tsay SH, et al. (2004) Acinar cell carcinoma of the pancreas: clinical and computed tomography manifestations. J Comput Assist Tomogr 28: 180-186. [Crossref]

13. http://surgpathcriteria.stanford.edu/pancreas/acinar_cell_carcinoma_pancreas/

14. La Rosa S, Adsay V, Albarello L, Asioli S, Casnedi S, et al. (2012) Clinicopathologic study of 62 acinar cell carcinomas of the pancreas: insights into the morphology and immunophenotype and search for prognostic markers. Am J Surg Pathol 36: 17821795. [Crossref]

15. Hashimoto M, Matsuda M, Watanabe G, Mori M, Motoi N, et al. (2003) Acinar cell carcinoma of the pancreas with intraductal growth: report of a case. Pancreas 26: $306-$ 308. [Crossref]

16. Fabre A, Sauvanet A, Flejou JF, Belghiti J, Palazzo L, et al. (2001) Intraductal acinar cell carcinoma of the pancreas. Virchows Arch 438: 312-315. [Crossref]

17. Basturk O, Zamboni G, Klimstra DS, Capelli P, Andea A, et al. (2007) Intraducta and papillary variants of acinar cell carcinomas: a new addition to the challenging differential diagnosis of intraductal neoplasms. Am J Surg Pathol 31: 363-370. [Crossref]

18. Klimstra DS, Heffess CS, Oertel JE, Rosai J (1992) Acinar cell carcinoma of the pancreas. A clinicopathologic study of 28 cases. Am J Surg Pathol 16: 815-837. [Crossref]

19. Basturk O, Zamboni G, Klimstra DS, Capelli P, Andea A, et al. (2007) Intraductal and papillary variants of acinar cell carcinomas: a new addition to the challenging differential diagnosis of intraductal neoplasms. Am J Surg Pathol 31: 363-370. [Crossref]

20. Cubilla AL, Fitzgerald PJ (1979) Cancer of the pancreas (nonendocrine): a suggested morphologic classification. Semin Oncol 6: 285-297. [Crossref]

21. Kong B, Cheng T, Qian C, Wu W, Steiger K, et al. (2015) Pancrease-specific activation of $\mathrm{m}$ TOR and loss of p53 induce tumors reminiscent of acinar cell carcinoma. Molecular Cancer 14: 212.

22. Abraham SC, Wu TT, Hruban RH, Lee JH, Yeo CJ, et al. (2002) Genetic and immunohistochemical analysis of pancreatic acinar cell carcinoma: frequent allelic loss on chromosome $11 \mathrm{p}$ and alterations in the APC/beta-catenin pathway. Am J Pathol 160: 953-962. [Crossref]

23. Défachelles AS, Martin De Lassalle E, Boutard P, Nelken B, Schneider P, et al. (2001) Pancreatoblastoma in childhood: clinical course and therapeutic management of seven patients. Med Pediatr Oncol 37: 47-52. [Crossref]

24. Murakami T, Ueki K, Kawakami H, Gondo T, Kuga T, et al. (1996) Pancreatoblastoma: case report and review of treatment in the literature. Med Pediatr Oncol 27: 193-197. [Crossref]

25. Lee JL, Kim TW, Chang HM, Lee SK, Kim MH, et al. (2003) Locally advanced acinar cell carcinoma of the pancreas successfully treated by capecitabine and concurrent radiotherapy: report of two cases. Pancreas 27: e18-22. [Crossref]

26. Cho KJ, Kim JY, Lee SS, Khang SK, Kim CW (1996) Mixed acinar-endocrine carcinoma of the pancreas--a case report. J Korean Med Sci 11: 188-192. [Crossref]

Copyright: (C2016 Ford J. This is an open-access article distributed under the terms of the Creative Commons Attribution License, which permits unrestricted use, distribution, and reproduction in any medium, provided the original author and source are credited. 